\title{
The signature of intestinal dysbiosis in inflammatory rheumatic diseases
}

\author{
Anca Cardoneanu ${ }^{1,2}$, Alexandra Maria Burlui 1,2, Elena Rezus ${ }^{1,2}$ \\ ${ }^{1}$ Department of Rheumatology, University of Medicine and Pharmacy "Grigore T. Popa” Iasi, Romania \\ ${ }^{2} 1^{\text {st }}$ Rheumatology Clinic, Clinical Rehabilitation Hospital lasi, Romania
}

\begin{abstract}
The study of intestinal microbiota is an important and current subject. It is well known that the gut microbiota plays a decisive role in the development of intestinal function, contributes to the defense against different infections, gives tolerance to ingested foods, regulating and maintaining the function of the intestinal barrier. The gut microbiota is different from individual to individual, determining, through the molecular profile, an "individual profile". Intestinal dysbiosis is associated with multiple diseases such as IBD, irritable bowel syndrome, nosocomial infections or rheumatic inflammatory disorders. By characterizing intestinal dysbiosis in patients, a link could be made between these bacteria and the pathogenic mechanisms of the diseases, assigning these structures key roles in the onset of systemic disorders. This allows a better understanding of the pathophysiological mechanisms of the diseases and allow having a targeted treatment aimed at improving dysbiosis and restoring the normal microbial gut profile.
\end{abstract}

Keywords: intestinal microbiota, dysbiosis, spondylarthritis

\section{INTRODUCTION}

Gut microbiota represents all the microorganisms in the community of microbes found in the gastrointestinal tract. The study of the intestinal microbiota is a current topic, and the new techniques of molecular biology and microbiology have allowed the identification of the diversity of bacterial species. Using PCR (Polymerase Chain Reaction), RT-PCR (Reverse Transcription-Polymerase Chain Reaction) or metagenomics (16S rDNA sequencing) analyzes, the gut microbiota was quantitatively evaluated and its role in the etiopathogenesis of various diseases could be estimated.

Each person presents, at the intestinal level, a set of relatively stable bacterial species that determines an ,individual profile" through the molecular signature. The intestinal tract of the newborn is sterile and the colonization begins with birth and diet (1). In contrast to adult microbiota, the newborn has a low bacterial diversity, dominated by Actinobacteria and Proteobacteria species (2). From the age of 1 year, each child has an individual bacterial profile, and af- ter the second year, the child's microbiota has the same composition as the microbiota of an adult person (3).

\section{INTESTINAL MICROBIOTA - STRUCTURE AND FUNCTIONS}

Currently, the composition of gut microbiota is well known, but its functions have not been fully elucidated. It includes between 500 and 1000 bacterial species divided into 2 major categories from phyla group: Bacteroides and Firmicutes. The number of bacterial species in the gut is about 10 times higher than the number of host somatic cells. Furthermore, the bacterial genome (2 million genes) is numerically superior compared to the human genome (4).

Gut microbiota plays an important role in the development of the intestinal immune system by modulating the formation of the intestinal mucosal layer and lymphoid structures, by the positive stimulating effect on the innate or adaptive immune system, by differentiating immune cells and producing immune 


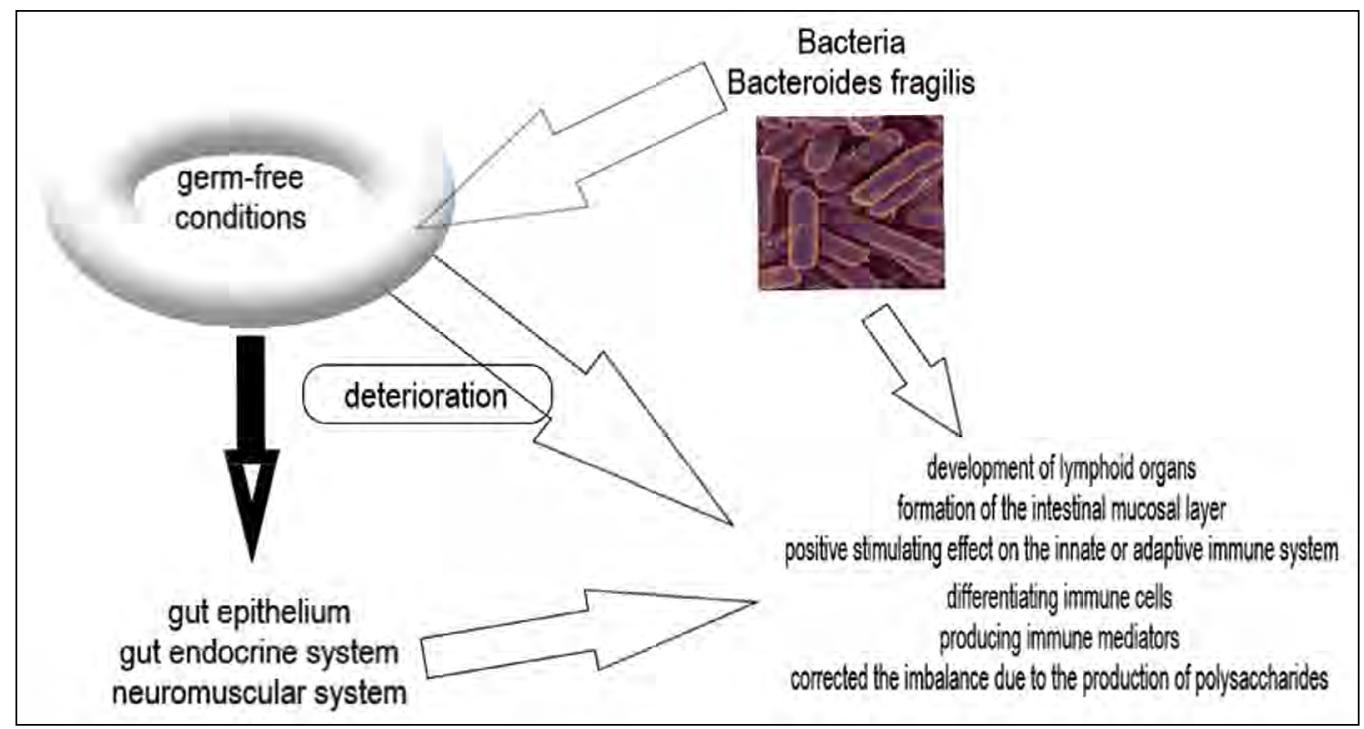

FIGURE 1. Modulation of intestinal microbiota by bacterial structures

mediators (5). Laboratory animal studies conducted under germ-free conditions have shown a deterioration of the intestinal epithelium, of the intestinal endocrine system, as well as of the neuromuscular system $(6,7)$. The introduction of Bacteroides fragilis into that sterile environment determined the development of lymphoid organs and corrected the imbalance due to the production of polysaccharides (8) (fig.1).

Intestinal bacteria have the ability to organize into stable and distinct groups. Researchers from 10 countries carried out the MetaHIT (Metagenomics of the Human Intestinal Tract) project, which analyzed the dynamics of bacterial diversity at the intestinal level (9). The 3 different studies included 145 European subjects and 154 American subjects. In all study groups, 3 bacterial enterotypes were identified: the $1^{\text {st }}$ enterotype - Bacteroides, the $2^{\text {nd }}$ enterotype - Prevotella, the $3^{\text {th }}$ enterotype - Ruminococcus (the most common). These bacterial enterotypes have been shown to be independent of the age of the subjects, their gender, body mass index or nationality. Each enterotype, characterized by functional differences and distinct bacterial clusters, reflects a different mode of energy generation, closely compatible with the host. Bacterial enterotypes have a permanent interaction with the host and have a strong impact on the health of the individuals.

There is a bacterial-host symbiosis highlighted by the results of studies published in the literature. The intestinal microbiota also has some metabolic roles. Thus, bacterial disaccharides cause fermentation of sugars, such as lactose, and their conversion into short-chain fatty acids. Of these, butyrate is considered to be a major source of energy for colonic epithelium, controlling the proliferation, growth and differentiation of intestinal epithelial cells (10). Some commensal bacteria participate in the metabolism of drugs such as sulfasalazine, other bacterial structures take part in the production of vitamins (vitamin $\mathrm{K}$, water soluble B vitamins, folic acid) or in the deconjugation of bile salts $(11,12)$.

The intestinal microbiota can also interact with the enteric nervous system, leading to changes in intestinal motility, sensory function and pain perception $(13,14)$. Due to the action on the development and functioning of the central nervous system, today we can talk about the existence of the microbiotabrain-gut axis $(15,16)$.

\section{INTESTINAL DYSBIOSIS AND RELATED DISEASES}

Dysbiosis is considered to be a change in the composition of the gut microbiota. It may be associated with alteration of host-microbiota interaction and immune system. Dysbiosis is characterized by an increased intestinal permeability and microbial translocation through the intestinal mucosa, which results in metabolic endotoxemia and systemic inflammation (numerous pro-inflammatory cytokines, oxygen free radicals) (17).

Recent studies have highlighted the association between intestinal dysbiosis and conditions such as nosocomial infections, necrotizing enterocolitis in premature babies, inflammatory bowel disease (IBD), irritable bowel syndrome, obesity, rheumatic autoimmune diseases or allergies. The pathogenesis 


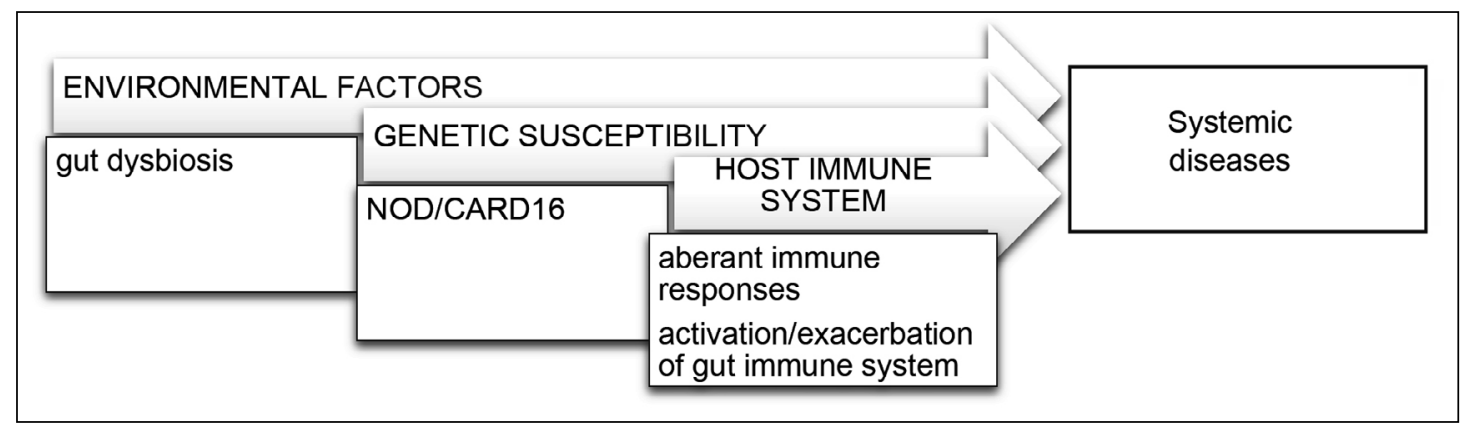

FIGURE 2. The main factors involved in the pathogenesis of systemic diseases starting from the alteration of the intestinal microbiota

of these disorders involves the interaction of several elements. The environmental factors, genetic susceptibility and immunity of the host form a triad that can regulate TCR (toll like receptor) function. Promoter environmental factors include bacterial antigens from the enteric microbiome. Genetic susceptibility mainly refers to the presence of the NOD/ CARD16 gene, which plays an important role in the recognition of microbial lipopolysaccharide. Alteration of the host's immune response may result in inappropriate activation or exacerbation of the intestinal immune system (17) (fig. 2).

Considering the above mechanisms, IBD can be considered the result of the interaction between genetic factors that confer susceptibility and environmental factors that influence the composition of the intestinal commensal microbial flora, thus leading to an abnormal mucosal response (18). Huttenhower et al. analyzed the synergy of genetic mechanisms and interaction with the gut microbiome in patients with IBD (19). Thus, 2 different processes such as autophagy and endoplasmic reticulum stress can function synergistically in the same cell type, favoring the emergence of a circumstance that promotes the disease. On the other hand, the same pathogenic mechanism may have different effects in different cells, which favors the appearance of IBD. An autophagy defect at the level of intestinal epithelial cells can affect Paneth cell function, while a similar macrophage autophagy defect influences interleukin-1 $\beta$ (IL-1 $\beta$ ) secretion, an important mediator in cell defense. IL$1 \beta$ participates in the modulation of the immune system and is closely related to the level of other pro-inflammatory cytokines such as IL-17 or IL-22 $(19,20)$.

\section{INTESTINAL DYSBIOSIS IN RHEUMATIC INFLAMMATORY DISEASES}

The close link between spondylarthritis and IBD is a well-known subject, these disorders being con- sidered distinct phenotypes of the same immunomediated disease. In both diseases, intestinal dysbiosis appears to play a key role, being an important factor in the evolution of these diseases, as well as in the treatment of patients.

Many studies have analyzed the epidemiological data regarding the association between inflammatory joint disease and IBD. Maeda et al. (21) reported 21 cases of arthritis (10.3\%) and 3 of ankylosing spondylitis (AS) $(1.5 \%)$ in a cohort of 203 Japanese patients diagnosed with Crohn's disease (CD). Triantafillidis et al. reported a 30\% frequency of arthritis / arthralgia in a group of 155 Greek patients with CD (22). The study by Bernstein (23) was based on the inclusion of Canadian patients with IBD, highlighting a $4 \%$ prevalence of $\mathrm{AS}$ in men with $\mathrm{CD}$, much more frequent than the group of patients diagnosed with ulcerative colitis (UC). Al-Hamali et al. reported a prevalence of arthritis of $8.9 \%$ in patients with UC in Kuwait, with a total frequency of rheumatic manifestations of approximately $31 \%$ (24).

Starting from this close connection between intestine and joints, the structure of the intestinal microbiome was analyzed both in patients with IBD and in those having peripheral or axial articular manifestations. The analysis was based both on highlighting bacterial structures and on analyzing the effect of dysbiosis on the immune system and on the production of pro-inflammatory factors.

Thus, patients having an active $\mathrm{CD}$ showed a significant decrease in DEFA5 and DEFA6 $\alpha$-defensins, which caused an impaired mucosal function and a modified commensal microbial flora $(25,26)$. Controversially, studies have shown an increased expression of $\alpha$-defensins in patients with AS who also had subclinical ileal inflammation (27). Depletion of the intestinal mucin layer leads to the formation of an inflammatory-like phenotype, to stress in the endoplasmic reticulum and an increasing in the pro- 
duction of IL-23 (28). Excess of IL-23 is sufficient to develop spondylarthritis in laboratory animal studies (29).

Regarding the IL-17/IL-22 proinflammatory cytokines relevant for IBD and spondylarthritis, clear evidence from murine studies indicates that the interaction between the intestinal microbiota and the host determines the activation of immune cells and the excess production of proinflammatory cytokines $(30,31)$.

Intestinal dysbiosis of patients with spondylarthritis is characterized by a decrease in microbial diversity, which is similar to what has been shown in patients with IBD. However, some results are contradictory depending on the method of analysis of the gut microbiota. Numerous clinical trials, which have used antibody tests, have shown an increase in Klebsiella species in patients diagnosed with AS (32). Another study, based on gel electrophoresis of faecal samples of patients with AS, did not reveal a statistically significant difference between these cases and healthy controls regarding the composition of the intestinal microbiota (33).

Recent studies, based on metagenomics and analysis of 16S rRNA sequencing, highlighted that intestinal dysbiosis in AS cases is characterized by a significant increase in populations of Paraprevotella and Bacteroides vulgatus (34). Also, the modification of the commensal intestinal flora represents a risk factor for the appearance of AS (34). Thus, the growth of the species of Lachnospiraceae, Ruminococcaceae, Rikenellaceae, Porphyromonadaceae and Bacteroidaceae, together with the decrease of the populations of Veillonellaceae and Prevotellace$a e$, constitutes a particular environment that favors the onset of autoimmune rheumatic diseases (fig.3) (34).

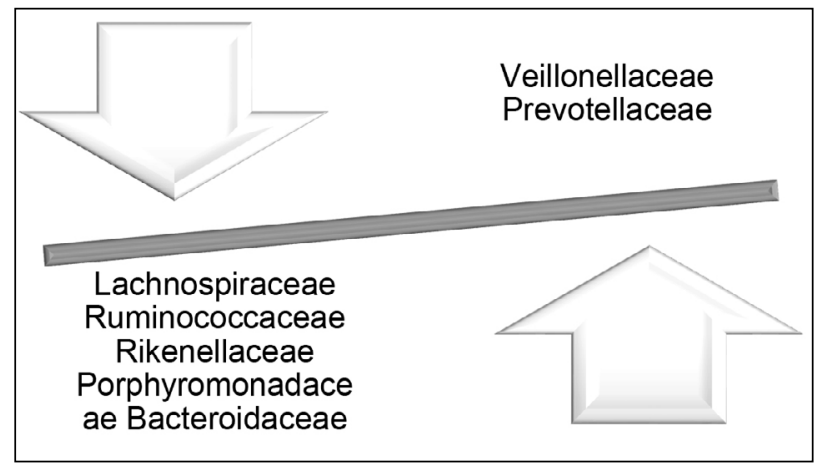

FIGURE 3. Intestinal dysbiosis in spondylarthritis

Tito et al. have analyzed the important role of intestinal dysbiosis, especially of the Dialister genus, in the pathogenesis of spondylarthritis (35). The study included 27 patients with spondylarthritis of whom 14 had microscopic intestinal inflammation and 13 had no intestinal inflammation. All patients underwent colonoscopy with ileal and colonic biopsies. 16S rRNA assay was used to compare the gut microbial composition. The results support the important role of intestinal dysbiosis in triggering spondylarthritis. Thus, the microbiota of patients with spondylarthritis was associated with intestinal inflammation, and the Dialister genus was positively correlated with the Ankylosing Spondylitis Disease Activity Score (ASDAS).

Recent studies support the fact that intestinal dysbiosis in patients with AS correlates with the presence of HLA-B27 antigen. 16S rRNA sequencing was used to detect HLA-B27-associated bacteria dysbiosis. Thus, numerically increased populations of Paraprevotella and Bacteroides vulgatus were associated with the predisposing genetic field (36).

The microbiota also plays an important role in the pathogenesis of other spondylarthritis such as psoriatic arthropathy, streptococcal infection being considered a trigger factor in genetically susceptible individuals (37). Recent studies using 16S rRNA assays have revealed significant differences between cutaneous microbiota of patients with vulgar psoriasis and healthy individuals, characterized by a decrease in staphylococci and Propionibacteria (38). In the intestinal microbiota, there was an increase in Firmicutes and a decrease in Actinobacteria and Proteobacteria compared to the control group (38).

Except for reactive arthritis in which there is a clear evidence that bacterial infections can cause joint manifestations, currently studies only present hypotheses regarding the role of the microbiota in the etiopathogenesis of immune-mediated arthritis (39). However, a murine HLA-B27 positive AS study highlighted that, in germ-free conditions, these animals did not develop joint inflammation $(39,40)$.

\section{INTESTINAL DYSBIOSIS - TREATMENT}

Medications commonly used for these diseases can change or modulate the gut microbiota, having clinically beneficial effects for the patient and improving bowel dysbiosis.

Prebiotics, oligofructose and inulin, had a positive effect on intestinal dysbiosis, leading to the growth of Lactobacillus and Bifidobacterium species (41). Prebiotics containing fermentable dietary 
fiber reduced intestinal inflammation by increasing the level of short-chain fatty acids $(42,43)$.

Probiotics, through the ability to modulate gut flora, have been compared to placebo in numerous clinical trials. The most investigated were Escherichia coli Nissle 1917 and VSL \# 3, a combination of Streptococcus, Bifidobacterium and Lactobacillus. The results were especially promising in patients diagnosed with UC. Thus, Kruis and his colleagues (44) demonstrated that the administration of Escherichia coli Nissle 1917 in cases of UC was associated with maintaining of clinical-biological remission, having an effect similar to that of salicylates. The efficacy of VSL \# 3 in inducing and maintaining UC remission, either alone or as an adjuvant treatment, was supported by the results of many clinical trials $(45,46)$.

The use of mesalazine has led to improvement of intestinal dysbiosis by reducing the concentration of adherent bacteria (47) and by decreasing the expression of genes associated with bacterial invasion (48). Thiopurines reduced the bacterial diversity in the faeces of patients with IBD (49). Anti-TNF $\alpha$ (Tumor necrosis factor $\alpha$ ) agents, successfully used in the treatment of IBD and spondylarthritis, have modulated the gut microbiota in these patients. Thus, there was a significant increase of Faecalibacterium prausnitzii, followed by the decrease of Escherichia coli species, after only 3 months of treatment with adalimumab (50).

\section{CONCLUSIONS}

The intestinal microbiota can be considered an „organ in an organ“, being assigned many functions of its own. The intestinal microbiota is in a continuous dynamic, being influenced by external factors such as: nutrition, infections, use of various drugs, geographical area of origin or psychological stress. Changes in the composition of the gut microbiota dysbiosis - is a new and attractive subject, being studied in numerous clinical trials. The data published in the literature support the hypothesis that intestinal dysbiosis plays an essential role, both in the pathogenesis of rheumatic inflammatory diseases and other systemic disorders.

Conflict of interest: none declared Financial support: none declared

\section{REFERENCES}

1. Margues TM, Wall R, Ross RP at al. Programming infant gut microbiota: influence of dietary and environmental factors. Curr Opin Biotechnol 2010;21:149-156.

2. Eckburg PB, Bik EM, Bernstein $\mathrm{CN}$ et al. Diversity of the human intestinal microbial flora. Science 2005;308:1635-1638.

3. Koenig JE, Spor A, Scalfone $\mathrm{N}$ et al. Succession of microbial consortia in the developing infant gut microbiome. Proc Natl Acad Sci USA 2011;108:4578-4585.

4. Guarner F, Malagelada JR. Gut flora in health and disease. Lancet 2003; 361: 512-519.

5. Akira S, Uematsu S, Takeuchi O. Pathogen recognition and innate immunity. Cell 2006;124:783-801.

6. Falk PG, Hooper LV, Midtvedt T, Gordon Jl. Creating and maintaining the gastrointestinal ecosystem: what we know and need to know from gnotobiology. Microbiol Mol Biol Rev 1998;62:1157-1170.

7. Uribe A, Alam M, Johansson $\mathrm{O}$ et al. Microflora modulates endocrine cells in the gastrointestinal mucosa of the rat. Gastroenterology 1994;107:1259-1269.

8. Mazmanian SK, Liu CH, Tzianabos AO, Kasper DL. An immunomodulatory molecule of symbiotic bacteria directs maturation of the host immune system. Cell 2005;122:107-118.

9. Arumugam M, Raes J, Pelletier E et al. Enterotypes of the human gut microbiome. Nature 2011;473:174-180.

10. David LA, Maurice CF, Carmody RN, et al. Diet rapidly and reproducibly alters the human gut microbiome. Nature 2014; 505:559-563.

11. Jones BV, Begley M, Hill C et al. Functional and comparative metagenomic analysis of bile salt hydrolase activity in the human gut microbiome. Proc Natl Acad Sci USA 2008;105:13580-13585.

12. Shanahan F. The host-microbe interface within the gut. Best Pract Res Clin Gastroenterol 2002;16:915-931.

13. Picard C, Fioramonti J, Francois A et al. Review article: Bifidobacteria as probiotic agents- Physiological effects and clinical benefits. Aliment Pharmacol Ther 2005;22:495-512.
14. Wang B, Mao YK, Diorio $C$ et al. Luminal administration ex vivo of a live Lactobacillus species moderates mouse jejunal motility within minutes. FASEB J 2010;24:4078-4088.

15. Konturek PC, Brzozowski T, Konturek SJ. Stress and the gut: pathophysiology, clinical consequences, diagnostic approach and treatment options. J Physiol Pharmacol 2011; 62: 591-599.

16. Cryan JF, O'Mahony SM. The microbiome-gut-brain axis: from bowel to behavior. Neurogastroenterol Motil 2011;23:187-192.

17. Melmed GY, Abreu MT. New insights into the pathogenesis of inflammatory bowel disease. Curr Gastroenterol Rep 2004; 6(6):474-481

18. Molodecky NA, Kaplan GG. Environmental risk factors for inflammatory bowel disease. Gastroenterol Hepatol (NY) 2010; 6(5): 339-346.

19. Huttenhower $C$, Kostic $A D$, Xavier RJ. Inflammatory bowel disease as a model for translating the microbiome. Immunity $2014 ; 40(6)$ : 843-854.

20. Coccia $M$, Harrison OJ, Schiering $C$ et al. IL-1 $\beta$ mediates chronic intestinal inflammation by promoting the accumulation of IL-17A secreting innate lymphoid cells and CD4(+) Th17 cells. J Exp Med 2012; 209(9):1595-1609.

21. Maeda K, Okada M, Yao T, et al. Intestinal and extraintestinal complications of Crohn's disease: predictors and cumulative probability of complications. J Gastroenterol. 1994; 29: 577-582.

22. Triantafillidis JK, Emmanouilidis A, Manousos O, et al. Clinical patterns of Crohn's disease in Greece: a follow-up study of 155 cases. Digestion. 2000; 61: 121-128.

23. Bernstein $\mathrm{CN}$, Blanchard JF, Rawsthorne $\mathrm{P}$, et al. The prevalence of extraintestinal diseases in inflammatory bowel disease: a population-based study. Am J Gastroenterol. 2001; 96: 1116-1122.

24. Al-Shamali MA, Kalaoui M, Patty I, et al. Ulcerative colitis in Kuwait: a review of 90 cases. Digestion. 2003; 67: 218-224.

25. Simms LA, Doecke JD, Walsh MD, et al. Reduced a-defensin expression is associated with inflammation and not NOD2 mutation status in ileal Crohn's disease. Gut 2008, 57:903-910. 
26. Wehkamp J, Salzman NH, Porter E, et al. Reduced Paneth cell a-defensins in ileal Crohn's disease. Proc Natl Acad Sci USA 2005, 102:18129-18134.

27. Ciccia F, Bombardieri M, Rizzo A, et al. Over-expression of paneth cell-derived anti-microbial peptides in the gut of patients with ankylosing spondylitis and subclinical intestinal inflammation. Rheumatology 2010, 49:2076-2083.

28. Heazlewood CK, Cook MC, Eri R, et al. Aberrant mucin assembly in mice causes endoplasmic reticulum stress and spontaneous inflammation resembling ulcerative colitis. PLoS Med 2008, 5:e54.

29. Sherlock JP, Joyce-Shaikh B, Turner SP, et al. IL-23 induces spondyloarthropathy by acting on ROR-gammat+ CD3+CD4-CD8entheseal resident T cells. Nat Med 2012, 18:1069-1076.

30. Salzman NH, Hung K, Haribhai D, et al. Enteric defensins are essential regulators of intestinal microbial ecology. Nat Immunol 2010, 11:76-82.

31. Shaw MH, Kamada N, Kim YG, et al. Microbiota-induced IL-1beta, but not IL-6, is critical for the development of steady-state TH17 cells in the intestine. J Exp Med 2012, 209:251-258.

32. Stone MA, Payne U, Schentag $C$, et al. Comparative immune responses to candidate arthritogenic bacteria do not confirm a dominant role for Klebsiella pneumonia in the pathogenesis of familial ankylosing spondylitis. Rheumatology 2004, 43:148-155.

33. Stebbings S, Munro K, Simon MA, et al. Comparison of the faecal microflora of patients with ankylosing spondylitis and controls using molecular methods of analysis. Rheumatology 2002, 41:1395-1401.

34. Costello ME, Ciccia F, Willner D, et al. Intestinal dysbiosis in ankylosing spondylitis. Arthritis Rheumatol 2015;67:686-691.

35. Tito RY, Cypers H, Joossens M, et al. Dialister as microbial marker of disease activity in spondyloarthritis. Arthritis Rheumatol. $2016 \mathrm{Jul}$ 7. doi: 10.1002/art.39802.

36. Lin P, Bach M, Asquith M, et al. HLA-B27 and human beta2microglobulin affect the gut microbiota of transgenic rats. PLoS One 2014;9:e105684.

37. Krueger JG. The immunologic basis for the treatment of psoriasis with new biolog ic agents. J Am Acad Dermatol 2002, 46:1-26.

38. Fahlen A, Engstrand L, Baker BS, et al. Comparison of bacterial microbiota in skin biopsies from normal and psoriatic skin. Arch Dermatol Res 2012, 304:15-22.
39. Jacques $P$, Elewaut $D$. Joint expedition: linking gut inflammation to arthritis. Mucosal Immunology. 2008; vol. 1, no. 5: 364-371.

40. Rudwaleit M, Baeten D. Ankylosing spondylitis and bowel disease. Best Pract Res Clin Rheumatol 2006; 20: 451-471.

41. Guarner F. Prebiotics in inflammatory bowel diseases. Br J Nutr. 2007; 98(Suppl 1):S85-S89.

42. Joo E, Yamane S, Hamasaki A, et al. Enteral supplement enriched with glutamine, fiber, and oligosaccharide attenuates experimental colitis in mice. Nutrition. 2013; 29(3):549-555.

43. Brotherton CS, Martin CA, Long MD, Kappelman MD, Sandler RS. Avoidance of fiber is associated with greater risk of Crohn's disease flare in a 6-month period. Clin Gastroenterol Hepatol. 2016; 14(8):1130-1136.

44. Kruis W, Fric P, Pokrotnieks J, et al. Maintaining remission of ulcerative colitis with the probiotic Escherichia coli Nissle 1917 is as effective as with standard mesalazine. Gut. 2004; 53(11):16171623.

45. Sood A, Midha V, Makharia GK, et al. The probiotic preparation, VSL\# 3 induces remission in patients with mild-to-moderately active ulcerative colitis. Clin Gastroenterol Hepatol. 2009;7:1202-1209.

46. Mardini HE, Grigorian AY. Probiotic mix VSL\#3 is effective adjunctive therapy for mild to moderately active ulcerative colitis: a metaanalysis. Inflamm Bowel Dis. 2014;20:1562-1567.

47. Andrews CN, Griffiths TA, Kaufman J, et al. Mesalazine (5-aminosalicylic acid) alters faecal bacterial profiles, but not mucosal proteolytic activity in diarrhoea-predominant irritable bowel syndrome. Aliment Pharmacol Ther. 2011; 34(3):374-383.

48. Kaufman J, Griffiths TA, Surette MG, et al. Effects of mesalamine (5-aminosalicylic acid) on bacterial gene expression. Inflamm Bowel Dis. 2009;15(7):985-996.

49. Wills ES, Jonkers DM, Savelkoul PH, et al. Fecal microbial composition of ulcerative colitis and Crohn's disease patients in remission and subsequent exacerbation. PLoS One. 2014; 9(3):e90981.

50. Busquets D, Mas-de-Xaxars T, Lopez-Siles M, et al. Antitumour necrosis factor treatment with adalimumab induces changes in the microbiota of Crohn's Disease. J Crohns Colitis. 2015; 9(10):899-906. 